Panel Cambio Climático, Ambiente y Biosfera

\title{
Niveles de Confort Térmico
}

\author{
P.I. Benito, D.F. Agnoli, M. Fontela, C. Murgo, L. Calivar, D. Caminos, I. Sattolo \\ Facultad de Informática, Ciencias de la Comunicación y Técnicas Especiales- Universidad de Morón \\ E-mail: pbenito@unimoron.edu.ar
}

\section{Resumen}

Para establecer los parámetros de diseño en el proyecto de instalaciones de calefacción, refrigeración y ventilación, se utilizan en general tablas 0 ábacos de Temperatura de Bulbo Seco, Humedad Relativa y Velocidad del Aire obtenidos por estudios experimentales realizados en países cuyos climas, formas de trabajo y tiempos laborales son diferentes. Por lo tanto, al utilizar estos parámetros se están adecuando nuestras instalaciones a otra realidad, dejando de lado los hábitos regionales, las características propias de nuestro clima y la adecuación cultural en cuanto a cómo se considera la gradualidad de los niveles de confort. El objetivo principal de esta investigación es determinar los Niveles de Confort Térmico regionales para condiciones ambientales, rangos horarios y tipos de actividad diferentes. La finalidad es optimizar el clima del ambiente de trabajo, a fin de lograr un estado de confort para el personal. Esto conlleva a una disminución de los riesgos laborales, aumentando el rendimiento y mejorando la calidad de vida. Esta investigación se realizó en una primera etapa para la Ciudad Autónoma de Buenos Aires (CABA) y Gran Buenos Aires. En una segunda etapa (que es la que nos ocupa), para la región de Cuyo. Las actividades consistieron en la selección de empresas e industrias representativas, relevamiento y análisis de datos para la construcción de gráficos de confort propios, como asimismo, brindar las recomendaciones de temperatura y humedad relativa, de acuerdo con el rango horario y actividad para la Región de
Cuyo. Además, y con el objeto de generalizar los resultados de esta investigación a cualquier región geográfica, se utilizaron programas destinados a la edición y evaluación de modelos gráficos probabilistas, para seleccionar un algoritmo que permita determinar el Nivel de Confort Térmico en función de la temperatura interior conociendo sus temperaturas exteriores, humedad relativa, velocidad de viento, latitud, longitud, tipo de actividad y horarios laborales. Los algoritmos seleccionados han sido contrastados con los datos de la Región de Cuyo. En una próxima etapa, se espera contrastar los datos aportados de cualquier región del país.

Palabras Claves: confort térmico, humedad relativa, temperatura, riesgo laboral, calidad de vida

\section{Introducción}

La búsqueda de los mejores niveles de confort laborales y la implementación de los medios para lograrlos es parte importante en la prevención del Riesgo Laboral. Generar un ambiente de trabajo adecuado para el desarrollo de las actividades, propiciándose un mayor bienestar a empleados y operarios, es una de las formas que permite reducir el riesgo laboral, aumentando asimismo el rendimiento personal y mejorando la calidad de vida.

\section{Antecedentes}

La ASHRAE (Sociedad Americana de los Ingenieros de Calefacción, Refrigeración y Aire Acondicionado), con la colaboración de diversas universidades y entidades 
oficiales realizó experiencias sobre las reacciones de distintas personas sometidas a diferentes condiciones de temperatura, humedad relativa y movimiento del aire. Estos resultados permitieron elaborar un diagrama de confort que relaciona las condiciones psicrométricas con la reacción humana.

El Método Fanger, publicado en la Revista Británica de Medicina Industrial en 1973 [1] valora el Confort Térmico en función del Voto Medio Estimado y el Porcentaje de Personas Insatisfechas. La estimación del Voto Medio se realiza en función de la aislación de la ropa, la Tasa Metabólica y las características del ambiente (Temperatura, Temperatura radiante, Humedad relativa y Velocidad del aire). Este índice permite determinar el porcentaje de personas insatisfechas con el ambiente.

El Método Fanger, denominado modelo fisiológico, fue desarrollado en cámaras controladas, con personas jóvenes en reposo y de origen europeo o norteamericano.

Por otro lado, están los Modelos Adaptativos (Humphreys, De Dear, Brager) [2] que tienen en cuenta el clima exterior para determinar las preferencias de confort interior, donde la persona no es un receptor pasivo, sino que junto al ambiente conforma un sistema dinámico.

Para establecer los parámetros de diseño en el proyecto de instalaciones de calefacción, refrigeración y ventilación, se utilizan en general tablas o gráficos de Temperatura de Bulbo Seco, Humedad Relativa y Velocidad del Aire obtenidos por métodos y experiencias realizados en países cuyos climas, formas de trabajo y tiempos laborales son diferentes.

Si bien, como se detalla en los antecedentes, se han realizado investigaciones sobre el tema, no se habían desarrollado experiencias en nuestro país, para las condiciones ambientales locales, ni se habían tenido en cuenta los rangos horarios, el tipo de actividad y la época del año.

\section{Estado actual del tema:}

Por ello, tomando como base estos modelos y considerando que los mismos no tienen en cuenta el tipo de actividad (trabajo real), los rangos horarios y las condiciones exteriores en función de las regiones climáticas, en simultaneidad con la reacción dinámica de la persona, se llevó a cabo una investigación sobre Niveles de Confort
Térmico Regionales para las distintas condiciones de trabajo en C.A.B.A y Buenos Aires (Benito y Agnoli , 2011) [3], que permitió brindar valores recomendados para la región, tipo de actividad, rango horario y época del año.

Asimismo, en el año 2012, el Grupo de Estudio Sobre Energía (G.E.S.E) de la Universidad Tecnológica Nacional Regional Pacheco presenta en el Seminario Nacional UTNRegional Mendoza un proyecto de investigación sobre "Análisis de Índices de Confort Térmico para las condiciones de la República Argentina, comenzando por C.A.B.A y la Provincia de Buenos Aires" (Borhi y otros, 2012) [4] en el que proponen la construcción de un mapa bioclimático que ofrezca índices para realizar balances térmicos óptimos, aportando datos actualizados de temperatura, humedad y velocidad del aire exteriores.

\section{Formulación del problema}

Para establecer los parámetros de diseño en los proyectos de instalaciones de calefacción, refrigeración y ventilación, se utilizan en general tablas o gráficos de temperatura de Bulbo Seco, Humedad Relativa y Velocidad del Aire obtenidos por estudios experimentales realizados en países cuyos climas, formas de trabajo y tiempos laborales son diferentes.

Al utilizar estos parámetros se están adecuando nuestras instalaciones a otra realidad, dejando de lado los hábitos regionales, las características propias de nuestro clima y la adecuación cultural en cuanto a cómo se considera la gradualidad de los niveles de confort.

Por ello, frente a este problema, las preguntas son: a) icuáles son los niveles de confort térmico específicos para nuestras condiciones laborales (rangos horarios / actividades) y climáticas de las Región de Cuyo?, b) iSe mejoran las condiciones de trabajo utilizando como parámetros de diseño los resultados de la investigación?

\section{Objetivos de la investigación}

- Determinar los niveles de confort térmico regionales para condiciones ambientales, rangos horarios y tipos de actividad diferentes en la Región de Cuyo, Argentina.

- Seleccionar las mejores condiciones de confort laboral en cuanto a temperatura, humedad relativa, velocidad del aire de acuerdo con el horario y actividad a desarrollar, a efectos de reducir el riesgo laboral. 
- Seleccionar un algoritmo que permita determinar el Nivel de Confort Térmico en función de la temperatura interior, conociendo la temperatura exterior, humedad relativa, velocidad de viento, latitud, longitud, tipo de actividad y horario laboral.

\section{Hipótesis}

- Los Niveles de Confort Térmico Regionales son específicos para cada actividad y rangos horarios de acuerdo con la región considerada en nuestro país.

- Los parámetros de los Niveles de Confort Térmico Regionales aplicados al cálculo de las instalaciones de Acondicionamiento del Aire reducen el riesgo laboral.

- Los parámetros de los Niveles de Confort Térmico Regionales aplicados al cálculo de las instalaciones de Acondicionamiento del Aire aumentan el rendimiento.

\section{Metodología}

- Relevamiento y Selección de Empresas

- Observación y Registro en dos etapas: PrimaveraVerano / Otoño- Invierno

- Clasificación y Cálculo Estadístico-Determinación de los parámetros.

- Confección de tablas y/o gráficos

- Comprobación de resultados

- Conclusiones y recomendaciones

\section{Actividades realizadas}

- Relevamiento de empresas e industrias en la región de Cuyo.

- Selección de las empresas y/0 industrias

- Registro de datos, a partir de una Encuesta de Confort Térmico

- Confección de gráficos en función de los datos obtenidos, por:

$$
\begin{aligned}
& \text { - Temporada del año } \\
& \text { - Horarios } \\
& \text { - Actividad }
\end{aligned}
$$

- Selección del algoritmo que permita determinar el Estado de Confort Térmico, teniendo en cuenta las siguientes variables:

- Temporada del año (Verano/Invierno)

- Horarios (Verano: 10-15- 18 hs- Invierno 10 hs.)
- Actividad (Leve- Moderado-Fuerte)

- Temperatura Interior

- Temperatura Exterior

- Humedad Relativa

- Velocidad del Viento

- Presión Atmosférica

- Latitud

- Longitud

Para la determinación del algoritmo, se decidió aplicar en este caso, Minería de Datos, que se define como un proceso de descubrimiento de nuevas y significativas relaciones, patrones y tendencias al examinar una gran cantidad de datos (Pérez López, 2007) [5]

El proceso consta de varias fases:

- Preparación de datos

- Exploración y auditoría

- Minería de datos propiamente dicha.

Evaluación del modelo con Elvira, programa destinado a la edición y evaluación de modelos gráficos probabilistas, concretamente redes bayesianas y diagramas de influencia [6]. Si se establecen rangos de temperaturas interiores, exteriores, porcentaje de humedad y velocidad del viento, fijando una variable, se pueden determinar el resto de las mismas (Fig. 1) 


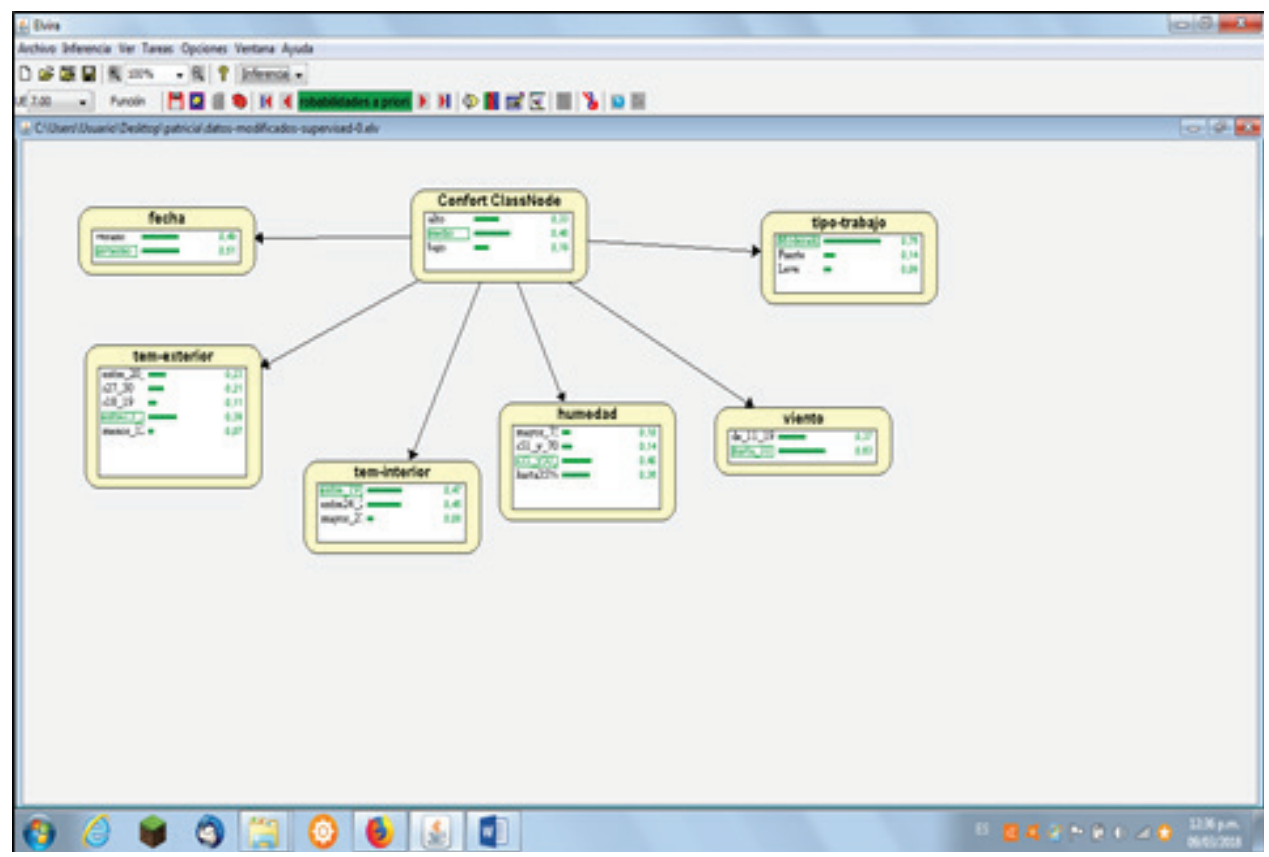

Figura 1. Evaluación del modelo con Elvira, programa destinado a la edición y evaluación de modelos gráficos probabilistas.

\section{Resultados y Conclusiones}

Los resultados obtenidos para la Región de Cuyo, tanto para verano como para invierno se muestran en la Tabla I.

Tabla I. Valores recomendados para la Región de Cuyo

\begin{tabular}{|c|c|r|}
\hline \multicolumn{3}{|c|}{ VERANO } \\
\hline Trabajo Físico & Temperatura & Humedad \\
\hline Leve & $27,0^{\circ} \mathrm{C}$ & $63,0 \%$ \\
\hline Moderado & $24,5^{\circ} \mathrm{C}$ & $52,0 \%$ \\
\hline Fuerte & $22,0^{\circ} \mathrm{C}$ & $60,0 \%$ \\
\hline
\end{tabular}

\begin{tabular}{|c|c|c|}
\hline \multicolumn{3}{|c|}{ INVIERNO } \\
\hline Trabajo Físico & Temperatura & Humedad \\
\hline Leve & $25,2^{\circ} \mathrm{C}$ & $46,8 \%$ \\
\hline Moderado & $24,0^{\circ} \mathrm{C}$ & $34,5 \%$ \\
\hline Fuerte & $23,0^{\circ} \mathrm{C}$ & $42,0 \%$ \\
\hline
\end{tabular}

Si se comparan estos resultados con los obtenidos en la investigación anterior: Niveles de Confort Térmico Regionales para las distintas condiciones de trabajo en C.A.B.A y Buenos Aires (Benito y Agnoli , 2011) (Tabla II), se observa, en el período de verano, que en la Región de Buenos Aires se logra el mismo grado de confort que en la Región de Cuyo, con una menor temperatura interior (en general difieren en $1^{\circ} \mathrm{C}$ ) al disminuir la humedad relativa. Para humedades relativas similares, las temperaturas interiores también lo son en correspondencia con el tipo de trabajo. En el período de invierno, se hace evidente que en la Región de Cuyo se requiere una mayor temperatura interior para humedades relativas similares, a efectos de obtener grados de confort comparables. Éstos resultados pueden deberse a la mayor variación de temperaturas exterior/interior en la Región de Cuyo respecto de Buenos Aires. 
Tabla II. Valores recomendados para la Región C.A.B.A. y Buenos Aires

\begin{tabular}{|c|c|c|}
\hline Trabajo Físico & Verano & Invierno \\
\hline Leve & $26^{\circ} / 46 \%$ & $21 \% / 45 \%$ \\
\hline Moderado & $25^{\circ} / 50 \%$ & $20 \% 55 \%$ \\
\hline Fuerte & $22 \% / 65 \%$ & $18,5 \% / 48 \%$ \\
\hline
\end{tabular}

De los resultados recomendados se puede inferir que, a menor humedad relativa, se requiere una menor temperatura interior para obtener estados de confort comparables. Si las variaciones de temperaturas exterior/ interior son muy pronunciadas, los estados de confort se obtienen aumentando las temperaturas interiores.

Por otra parte, el algoritmo seleccionado ha sido contrastado con los datos de la Región de Cuyo. En una próxima etapa, se espera contrastar los datos aportados de cualquier región del mundo.

\section{Bibliografía}

[1] Fanger, P., (1973), Assessment of man's thermal comfort in practice. in British Journal of Industrial Medicine. Recuperado de https://www.ncbi.nlm.nih.gov/

[2] Humphreys, M , Developing an Adaptive Model of Thermal Comfort and Preference. Recuperado de www. cbe.berkeley.edu.

[3] Benito, P. y Agnoli (2011), Niveles de Confort Térmico, en Revista de Seguridad. Año LXX-Nº 410, pp 26-29.

[4] Bohri, J. et al, (2012), Análisis de Índices de Confort Térmico para las condiciones de la República Argentina, comenzando por C.A.B.A y la Provincia de Buenos Aires. Recuperado de http://www.edutecne.utn.edu.ar/energia ure_mendoza_2012/01-analisis-FRGP.pdf

[5] Pérez López, C.,(2007), Minería de datos: técnicas y herramientas, Editorial Paraninfo.

[6] http://www.ia.uned.es/ fjdiez/bayes/elvira/manual/ manual.html

\section{Bibliografía adicional}

- Frank, E. et al, ( 2016), The Weka WorkBench, Online Appendix for "Data Mining: Practical Machine Learning Tools and Techniques", Morgan Kaufmann, Fourth Edition.

- Quadri, N., (2007), Instalaciones de Aire Acondicionado y Calefacción, Bs.As. Ed. Alsina. $8^{\circ} \mathrm{Ed}$.

- Serrano, J., Manual de Aire Acondicionado y Ventilación Industrial. Tomo 2 ebook: http://calderazi.com.ar/tag/ vmg-studio/

- www.insht.es/InshtWeb/Contenidos/Documentacion/.../ ntp_074.pdf

- http://www2.asepeyo.es/apr/apr0301.nsf/ficheros/ HAF0505009\%20Confort\%20T\%C3\%A9rmico.pdf/\$file/ HAF0505009\%20Confort\%20T\%C3\%A9rmico.pdf

- $\quad$ https://riunet.upv.es/bitstream/handle/10251/13751/ PROYECT0\%2OFINAL\%20DE\%20GRAD0.\%20Laura\%20 Solana\%20Mart\%C3\%ADnez.pdf?sequence $=1$

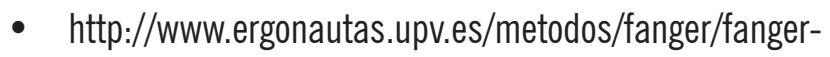
ayuda.php

- http://www.edutecne.utn.edu.ar/energia_ure_ mendoza_2012/01-analisis-FRGP.pdf

- http://www.cs.waikato.ac.nz/ml/weka/citing.html

- http://www.alcula.com/es/calculadoras/estadistica/ diagrama-de-caja 